\title{
Object Categories and Expertise: Is the Basic Level in the Eye of the Beholder?
}

\author{
James W. TANaKa AND Marjorie Taylor \\ University of Oregon
}

\begin{abstract}
Classic research on conceptual hierarchies has shown that the interaction between the human perceiver and objects in the environment specifies one level of abstraction for categorizing objects, called the basic level, which plays a primary role in cognition. The question of whether the special psychological status of the basic level can be modified by experience was addressed in three experiments comparing the performance of subjects in expert and novice domains. The main findings were that in the domain of expertise (a) subordinate-level categories were as differentiated as the basic-level categories, (b) subordinate-level names were used as frequently as basic-level names for identifying objects, and (c) subordinatelevel categorizations were as fast as basic-level categorizations. Taken together, these results demonstrate that individual differences in domain-specific knowledge affect the extent that the basic level is central to categorization. (1) 1991 Academic. Press, Inc.
\end{abstract}

In a series of important experiments. Rosch. Mervis, Gray, Johnson, and Boyes-Braem (1976) established that a basic level of abstraction has special significance in human categorization (also see Brown, 1958). The basic level was shown to be the most inclusive level at which a generalized shape of category exemplars is identifiable and imaginable. In addition, basic categories elicit similar motor programs and basic-level category labels are the first names learned by children. Based on their analysis of structure at the basic level, Rosch et al. (1976) predicted that basic-level categories would be the classifications made when objects are first perceived.

Rosch et al. (1976) demonstrated the special status of basic-level categories for object identification in a free-naming and a categoryverification task. In the naming task, subjects were presented with a series of pictures in rapid succession and were asked to write down the

We would like to thank our subjects for their enthusiastic support of this project, Gregory Murphy for his expert advice, Robert Mauro for the use of his lab, and Steve Jones, Keith Millis, Lisa Arnold, Todd Bennett, and Athena Wang for their assistance with Experiment 1. We would also like to thank Fred Attneave, Asher Cohen, Oliver John, Peter Jusczyk, and Mick Rothbart for comments on an earlier draft of this paper. We are also grateful to Douglas Medin and two anonymous reviewers for their helpful reviews of the manuscript. Correspondence concerning this article should be addressed to James Tanaka who is now at the Department of Psychology, Severance Laboratory, Oberlin College, Oberlin, OH 44074. 
word which named the object. The main finding was that subjects used basic-level names (e.g., table, bird) more frequently to identify objects than superordinate-(e.g., furniture, animal) or subordinate-level names (e.g., coffee table, robin). In the category-verification task, subjects heard a category label (superordinate, basic, or subordinate) and then indicated whether a picture shown after a brief delay was an exemplar of the category. The results showed that subjects were faster to categorize objects at the basic level than at the superordinate or subordinate levels. Rosch et al. interpreted subjects' naming preferences and verification times as indicating that people first identify objects at the basic level and then access the superordinate or subordinate categories.

An important issue raised by the research of Rosch and her colleagues is the extent to which the basic level is determined by structure in the world or in the mind of the perceiver. As Rosch et al. (1976) and others (Malt \& Smith, 1984; Mervis \& Rosch, 1981) have pointed out, attributes are not distributed randomly across objects in the world, but instead typically occur in correlated clusters. For example, feathers and wings occur together more frequently than fur and wings. According to Rosch (1978), "a working assumption of the research on basic objects is that (1) in the perceived world, information-rich bundles of perceptual and functional attributes occur that form natural discontinuities, and that (2) basic cuts in categorization are made at these discontinuities" (p. 31). It is important to note that, although Rosch's research emphasizes structure in the world, she did not view this structure as existing independently of the human perceiver. Rosch (1978) was careful to explain that it is the interaction between the human perceiver and the world that specifies the basic level. Lakoff (1987) also describes basic-level category formation as a consequence of human perceiver and object-world interactions. "Perhaps the best way of thinking about basic-level categories is that they are 'human-sized.' They depend not on the objects themselves, independent of people, but on the way people interact with objects: the way they perceive them, image them, organize information about them and behave toward them with their bodies" (p. 51).

In addition to the general role of the human perceiver, Rosch et al. (1976) speculated that individual differences in domain-specific knowledge could also be important in determining the basic level. For example, they found that one of their subjects who was an airplane mechanic answered questions about airplanes quite differently from the other subjects. Based on this observation, Rosch et al. (1976) suggested that the contribution of the perceiver to the categorization process could be cxamined by research which systematically varied subjects' level of expertise with respect to the objects being categorized. 
Although a number of studies have examined differences between experts and novices in pattern recognition (Biederman \& Shiffrar, 1988; Chase \& Simon, 1973) and conceptual organization (e.g., Chi, Feltovich, \& Glaser, 1981; Murphy \& Wright, 1984; Schvaneveldt, Durso, Goldsmith, Breen, \& Cooke, 1985), the issue of whether there is a change in the structure of classification hierarchies in object categorization with expertise has not been addressed. This question has become particularly important given the recent interest in how a person's knowledge in a given domain affects their conceptual structure (Carey, 1985; Medin \& Ortony, 1989; Murphy \& Medin, 1985). Neisser (1987) describes the emphasis of categorization research as shifting from the role of objective characteristics of objects to the role of people's theories and knowledge about those objects. According to Neisser, research based solely on the characteristics of objects will never provide an adequate account of categorization.

In the present experiments, we examined the performance of subjects in expert and novice knowledge domains on three tasks: feature listing, object naming, and category verification. Previously, subjects' feature lists have been used to identify the basic level (Rosch et al., 1976). Subjects list many more attributes for basic-level categories than for superordinate-level categories and do not add many new attributes for subordinate-level categories. However, experts and novices differ in their knowledge about subordinate category attributes, and this difference should be reflected in their feature lists. Thus, in Experiment 1, we examined subjects' feature lists for categories in expert and novice domains. We hypothesized that the usual patterns in feature lists reported by Rosch et al. (1976) might be altered when subjects are experts in the domain. More specifically, we expected that experts would list at least as many new features for subordinate-level categories as for basic-level categories.

In Experiments 2 and 3, we tested the effects of expertise on object categorization. As mentioned above, subjects tend to supply basic-level labels when asked to name objects, and they are fastest to verify category membership at the basic level. The primacy of the basic level in naming and category-verification tasks has been interpreted as a consequence of the differentiation of basic-level categories (i.e., the structure to be found in subjects' attribute lists). However, if experts know many attributes that distinguish objects at the subordinate level, they might be more apt than novices to identify objects at the subordinate level, rather than at the basic level. For example, a bird expert might spontaneously identify a bird with its subordinate-level name (e.g., "robin") instead of its basiclevel name (e.g., "bird"). The increased differentiation of subordinatelevel categories should also affect the speed at which experts are able to verify subordinate category membership. 


\section{EXPERIMENT 1}

\section{Feature Listing}

Most operational definitions of the basic level refer to the attribute structure in classification hierarchies. Rosch et al. (1976) found that subjects listed significantly more attributes for basic-level categories (e.g., hammer, chair, car) than for superordinate categories (e.g., tool, furniture, vehicle). In addition, the number of new attributes added for subordinate-level categories (e.g., ball peen hammer, kitchen chair, sedan) were significantly fewer than the number added for the basic-level categories. Rosch et al. (1976) noted that the effect of expertise was not examined in their research, and they speculated about its possible effect on the location of the basic level. "Would, for example, an ichthyologist, whether presented with an actual example of a category or with a fish name, been able to list sufficient attributes specific to trout, bass, and salmon that the basic level for fish would have been placed at that level of abstraction?'' (p. 393).

Rosch et al.'s speculation suggests that the number of features added at the subordinate level should increase as a consequence of expertise in a domain. Note, however, that one might not expect similar increases in the number of attributes listed at the basic or superordinate level as a function of expertise. Presumably, much of the knowledge that makes a person an expert concerns the subordinate categories in the domain. For example, the expert birdwatcher knows more than the novice with respect to the characteristics of specific kinds of birds (e.g., robins, sparrows) and in fact, may not necessarily be more knowledgeable about the general characteristics that distinguish birds from other kinds of animals. Thus, we predicted that an expert's knowledge would be demonstrated by an increase in the number of attributes listed for subordinate-level categories rather than by an increase in attributes distributed equally throughout the hierarchy.

To test this hypothesis, we asked bird and dog experts to list features for superordinate, basic, and subordinate categories. We describe a feature as new for a particular level if it is not listed at a more inclusive level of abstraction. For example, the property "has wings" is a new feature for the basic category "bird" because it is not a feature for the more general superordinate category "animal." However, the same feature "has wings" is not new for the subordinate category "robin" because it is also true of the more inclusive category "bird." If expert knowledge

\footnotetext{
${ }^{1}$ The new feature index is equivalent to the method employed by Rosch et al. (1976). In their feature-listing study, they found that subjects listed slightly more features for subordinate-level categories than for basic-level categories. However, the number of new features
} 
is added primarily at the subordinate level of categorization, experts should list as many new features for subordinate-level categories as for basic-level categories. In the novice domain, however, subjects should list more new features for the basic-level category than for subordinatelevel categories. Thus, we predicted an interaction between knowledge domain (expert and novice) and category level (basic and subordinate) for the number of new features listed by dog and bird experts.

\section{Method}

Subjects. Twelve dog and 12 bird experts participated in the experiment. Subjects were selected on the basis of their membership and participation in local dog and birdwatching organizations and on the basis of personal recommendations from other organization members. All subjects had a minimum of 10 years experience in their area of expertise with the majority of experts having over 20 years of experience. The group of dog experts consisted of nine women and three men ranging in age from 40 to 70 years with a mean age of 50 years. The group of bird experts consisted of five women and seven men ranging in age from 32 to 76 years whose mean age was also 50 years. Subjects were paid for their participation.

Prior to the feature-listing task, subjects filled out a general questionnaire concerning their past experience with dog and bird animals, membership in related professional organizations, and subscriptions to professional journals and magazines. Analysis of the questionnaire responses indicated that the two groups of experts met the criteria of expertise for cither the dog or bird domains. None of the subjects had extensive experience in both domains.

Stimuli. The subordinate-level bird categories selected for the feature-listing experiment were "robin," "crow," "jay," and "cardinal." The subordinate-level dog categories were "beagle," "Doberman pinscher," "collie," and "poodle." The four bird exemplars were among the 10 most frequently mentioned birds in Battig and Montague's (1969) category study. The four dog exemplars were among the 20 most frequently occurring dogs as determined by the American Kenuel Club's list of registered dogs for 1984. (The category of dog was not included in the Battig and Montague study.) Subjects also completed feature listings for the superordinate category "animal" and basic categories "dog" and "bird." Additional categories were used as fillers: "fruit," "musical instrument," and "furniture" (superordinate level); "chair" and "table" (basic level); "easy chair," "desk chair," "coffee table," and "kitchen table" (subordinate level). In total, there were 20 categories: 11 categories from the two expert domains and 9 filler categories. Category names were printed at the top of separate sheets of $81 / 2 \times 11^{\prime \prime}$ paper and randomly assembled into test booklets with the restriction that categories sharing the same superordinate could not appear in consecutive presentations.

Procedure. At the beginning of the experiment, subjects were given the following written instructions: "At the top of each attached sheet of paper is the name of a familiar object. In the spaces provided, list as many characteristics or attributes that you can think of which describe the object. For example, for the common object 'fish,' you might list the characteristics of 'swims,' 'lives in water,' 'has gills,' etc. The same characteristic can be listed for

added at the basic level were significantly more than the number of new features added at the subordinate level. Thus, by Rosch et al.'s definition, the basic-level category is determined by the amount of information gained when moving from a more general level of abstraction to a more specific level. 
more than one item. You will have two minutes for each item. The experimenter will signal when it is time to turn the page and go on to the next item. Remember to list only those things that arc characteristic of the object and avoid listing simple free associations (e.g., 'salt' and 'pepper')." Subjects were tested individually.

\section{Results and Discussion}

Differentiation of subordinate level categories. The number of features added at the basic and subordinate levels was determined for each individual subject based on his or her own feature list. Features that were semantically equivalent (e.g., "big" and "large") were collapsed and scored as a single feature as decided by a panel of three judges. To obtain the number of new features added at each level of abstraction, features were deleted from the more specific level of abstraction if the subject listed the same feature at a more inclusive level. For example, if a subject listed the attribute "has wings" for the basic level of "bird" and for the subordinate-level category "robin," it was deleted from the subordinatelevel category. A single new features value for the subordinate-level categories was obtained by averaging the number of new features for the four subordinate categories (e.g., "cardinal," "crow," "jay," "robin"). Thus, after removing any repeated or synonymous features from the feature lists, the number of new features for the superordinate-, basic-, and subordinate-level categories was obtained for each individual subject.

For categories outside the domain of expertise (e.g., bird categories for dog experts), we expected to replicate the finding that subjects list more new features for basic-level categories than for subordinate-level categories. However, we predicted that representations of subordinate categories in the domain of expertise would contain as many new features as their basic-level equivalents. To test this prediction, an ANOVA was performed with expert type (bird expert and dog expert) as a betweengroup factor and category level (basic and subordinate) and knowledge domain (expert and novice) as within-subject factors. The main effect for expert type was not significant, $F(1,22)=.75, M S e=22.28$, ns. However, experts listed more features in their knowledge domain of expertise, $F(1,23)=6.45, M S e=17.72, p<.05$, and the number of features was affected by category level, $F(1,23)=27.59, M S e=207.83, p<.001$. As predicted, the interaction between category level and knowledge domain was significant, $F(1,23)=19.82, M S e=68.77, p<.001$. As shown in Fig. 1, the source of the interaction can be attributed to the increased number of attributes listed at the subordinate level in the domain of expertise. That is, in the expert knowledge domain, subjects added almost the same number of attributes at the subordinate level as at the basic level. Hence, the bird expert knows as much about the distinguishing properties of "robin" and "crow" as he or she knows about the 


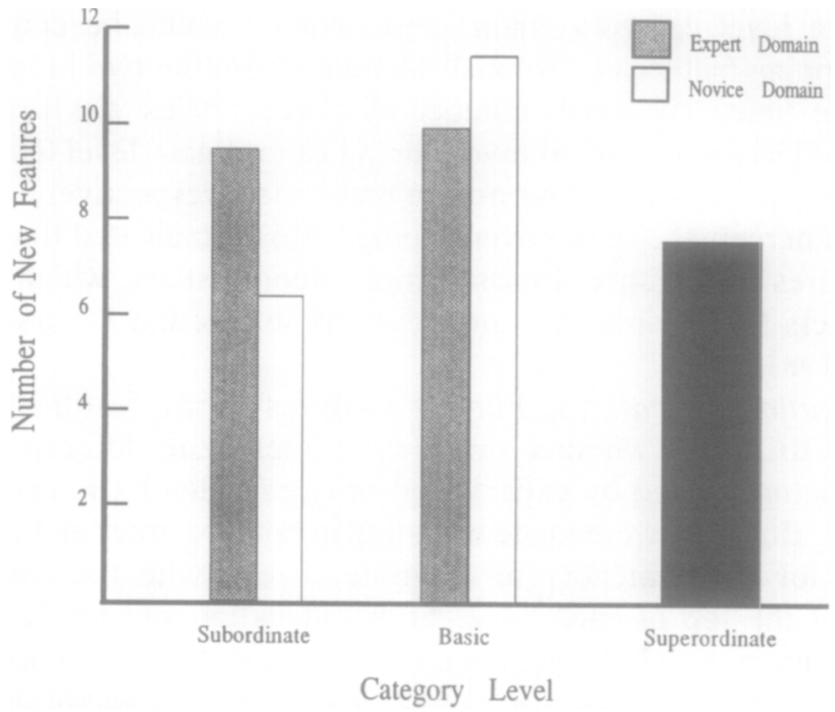

Fig. 1. Mean number of new features listed by subjects as a function of knowledge domain (expert and novice) and category level (subordinate, basic, and superordinate). Note that the basic-level categories "bird" and "dog" share the same superordinate category "animal."

distinguishing properties of "bird." However, consistent with the original Rosch et al. result, in the novice knowledge domain, subjects were able to list substantially more attributes at the basic level than at the subordinate level.

The findings reported above suggest that expert knowledge is primarily organized at the subordinate level of abstraction rather than at the basic level. In addition to the interaction between knowledge domain and category level with respect to new features, we were interested in (a) the extent that features listed for one subordinate category overlapped with features listed for the other subordinate level categories, and (b) the possibility that experts and novices differed in the kinds of attributes they listed at the basic and subordinate levels.

Degree of feature overlap at the subordinate level. By calculating the number of nonoverlapping subordinate features, it was possible to determine if subjects simply described subordinates at a finer level of detail or if they added information that was distinctive to particular subordinatelevel categories. Two independent judges scored each feature listed by each subject as "nonoverlapping" if it was listed for only one of the subordinate-level categories or as "overlapping" if it was listed for more than one category. For example, if a subject listed the feature "red breast" for the subordinate-level category "robin" and not for any other 
subordinate level category, then "red breast" would be considered a nonoverlapping feature of "robin." The data from the two judges agreed 98\% of the time. The small number of discrepancies was resolved by discussion. The amount of nonoverlapping subordinate-level features was 75 and $74 \%$ for the expert and novice categories, respectively. The relatively high percentage of nonoverlapping features indicated that subjects listed features with respect to some implicit contrast set, which appeared to be objects that shared the same level of abstraction (Tversky \& Hemenway, 1984). ${ }^{2}$

Classification of features. Further analyses of the feature lists were performed to assess whether there were interesting differences in the types of features listed by experts and novices at the basic and subordinate levels. Booklets were made by listing in random order all the features generated for each category on separate sheets, with the name of the category at the top of each page. Four judges independently classified individual features as belonging to one of four categories: (1) behaviorseither an action (e.g., barks) or habitat (e.g., lives in trees) of the animal, (2) parts-a segment or portion of the animal (e.g., eyes, beak), (3) dimension-the color or size of the animal, and (4) none of the above. For part features, judges were told to indicate whether the part was simply named (e.g., ears) or contained additional information (e.g., floppy ears). If three of the four judges agreed on the classification of the feature, it was added to a final master list. To find the number of behaviors, parts, and dimensions listed by each subject, the original feature lists were scored according to the master list. Thus, for each subject, the number of behaviors, parts, and dimensions mentioned for the basic- and subordinatelevel categories was obtained. For the subordinate level, a single value was calculated by averaging the number of features of each type across the four subordinate level categories. Three separate ANOVAs were performed for the three feature categories, with expert type (bird expert or dog expert) as a between-groups factor, and category level (basic or subordinate) and knowledge domain (expert or novice) as within-subjects factors.

\footnotetext{
${ }^{2}$ Murphy and Wright (1984) found that the categories of expert clinical psychologists were in fact less distinctive than the categories of intermediate and novice psychologists (child counselors and undergraduate students). There are several explanations that could account for the discrepancy between our result and Murphy and Wright's. Perhaps, the subordinatelevel categories selected for our study happened to be more distinctive than the psychological categories used in the Murphy and Wright experiment. Alternatively, as mentioned by Murphy and Wright (1984), there may be important differences between the structure of abstract categories, such as those used in psychological assessment, and the structure of object categories (Barsalou, 1985) as well as differences in how experts organize abstract categories versus object categories.
} 
Behaviors. This analysis yielded main effects for knowledge domain, $F(1,22)=4.23, M S e=7.09, p<.05$, and category level $F(1,22)=14.01$, $M S e=48.76, p<.01$, as well as a significant interaction between these two factors, $F(1,22)=5.60, M S e=10.72, p<.05$. However, these effects should be interpreted in light of the significant three-way interaction between expert type, knowledge domain, and category level, $F(1,1,22)=17.96, M S e=34.34, p<.01$. As shown in Table 1, in the category of dog, significantly more behaviors were listed at the basic level than at the subordinate level by both dog experts, $t(12)=2.87, p<.05$, and novices, $t(12)=2.40, p<.05$. However, in the bird category, experts listed slightly more behaviors at the subordinate level than at the basic level. Bird novices, on the other hand, listed significantly more behavioral features at the basic level than at the subordinate level, $t(12)=9.62, p<$ .01. These results indicate that novices list behaviors primarily at the basic level. Moreover, behavioral features do not distinguish dog experts and novices, but do distinguish bird experts and novices. This finding suggests that attending to behavioral cues (e.g., habitat, feeding activity) is a particularly important aspect of bird expertise.

Parts. There was a significant main effect for category level, $F(1,22)=$ 6.86, $M S e=31.80, p<.05$, showing that subjects listed more parts for basic-level categories than for subordinate-level categories, as Tversky and Hemenway (1984) found in their research. However, as shown in Table 1, there was a significant three-way interaction between expert type, knowledge domain, and category level, $F(1,1,22)=4.82, M S e=$ $13.69, p<.05$. In the bird category, both experts, $t(12)=3.42, p<.01$, and novices, $t(12)=2.21, p<.05$, listed significantly more parts at the basic level than at the subordinate level. However, in the dog category, novices listed significantly more parts at the basic level, $t(12)=3.16, p<$

TABLE 1

Means and Standard Deviations (in Parentheses) of New Features Listed as a Function of Object Category, Expertise, Feature Type, and Category Level

\begin{tabular}{|c|c|c|c|c|}
\hline & \multicolumn{2}{|c|}{ Dog category } & \multicolumn{2}{|c|}{ Bird category } \\
\hline & Experts & Novices & Experts & Novices \\
\hline \multicolumn{5}{|c|}{ Behavioral features } \\
\hline Subordinate & $2.50(.84)$ & $2.00(1.47)$ & $5.06(1.99)$ & $3.15(1.00)$ \\
\hline Basic & $4.91(2.81)$ & $4.83(3.75)$ & $4.17(2.12)$ & $4.50(1.98)$ \\
\hline \multicolumn{5}{|l|}{ Part features } \\
\hline Subordinate & $2.96(1.34)$ & $2.25(1.07)$ & $1.44(.92)$ & $1.33(.91)$ \\
\hline Basic & $2.50(3.43)$ & $3.50(1.88)$ & $3.41(1.93)$ & $3.17(2.76)$ \\
\hline \multicolumn{5}{|c|}{ Dimensional features } \\
\hline Subordinate & $2.29(.87)$ & $1.38(.53)$ & $1.54(.66)$ & $1.40(.67)$ \\
\hline Basic & $.25(.45)$ & $.42(.67)$ & $.42(.67)$ & $.50(.67)$ \\
\hline
\end{tabular}


.01 , but experts listed slightly more parts at the subordinate level. Thus, the amount of part information listed at the basic and subordinate levels distinguished dog experts and novices, but not bird experts and novices.

Part features were further analyzed in terms of the number of modified part features. At the basic level, only $14 \%$ of the parts were modified whereas at the subordinate level, $78 \%$ were modified. This finding is consistent with Tversky and Hemenway's (1984) result showing that unmodified parts are most frequently listed at the basic level and modified parts at the subordinate level. With respect to expertise, in the dog category the mean number of modified parts listed by experts and novices was not significantly different, 2.5 and 2.2 , respectively. However, in the bird category, there was a significant difference, $t(12)=2.35, p<.05$, between the mean number of modified parts listed by bird experts, 1.25, and novices, .58 . Bird experts listed the same number of subordinate level parts as the novices, but significantly more of these were modified parts.

Dimensions. Overall, experts listed more dimensional features than novices, $F(1,22)=4.50, M S e=.99, p<.05$, and more dimensional features were listed at the subordinate level than at the basic level, $F(1,22)$ $=68.18, M S e=37.81, p<.001$. The main effect for expert type was not significant, $F(1,22)=1.58, M S e=.77, n s$, and expert type was not involved in any significant interactions. There was a significant interaction between knowledge domain and category level, $F(1,22)=5.10, M S e$ $=2.58, p<.05$. As shown in Table 1, both experts and novices listed very few dimensions at the basic level. At the subordinate level, more dimensions were listed and at this level, experts listed more dimensional features than novices, $t(23)=2.73, p<.05$.

\section{Summary}

According to Rosch et al. (1976), a defining characteristic of a basiclevel category is that it contains a larger number of new features than either its superordinate- or subordinate-level categories. We found that in their domain of expertise, subjects listed almost as many new features for subordinate-level categories as the basic-level category. Thus, our results show that the distinctiveness of subordinate-level categories changes as a function of expertise.

The above analyses also clarified the type of knowledge that distinguishes experts from novices. The exact nature of expertise varies across domains, and thus, the object attributes that experts select as most salient will depend on the goals and demands of the task domain. For example, we would expect that because the goals of expert ichthyologists and expert sport fishermen are different, their knowledge of fish would also differ in certain ways. Similarly, we found that differences exist between what bird experts and dog experts list as the salient features of the animals 
in their domain. Dog experts differed from novices in listing more part features, whereas bird experts differed from novices in listing more modified part features. Bird experts listed more behavioral features than novices. Both dog and bird experts listed more dimensional features than novices. With respect to the more general issue of expertise and category structure, it was found that in both these domains, the experts' knowledge was added at the subordinate level of abstraction. One implication of this information increase at the subordinate level is that subordinate-level categories may play a more central role in the processes of object categorization in an expert domain. In the next two experiments, the effect of expertise on object categorization is more closely examined.

\section{EXPERIMENT 2}

\section{Free Naming Study}

Previous researchers (Jolicoeur, Gluck, \& Kosslyn, 1984; Rosch et al., 1976; Segui \& Fraisse, 1968) have shown that subjects use basic-level names (e.g., bird, dog, chair, hammer) when asked to spontaneously identify pictures of common objects. This finding has been used as evidence that the most accessible level of abstraction for categorizing objects is the basic level. However, given the increased differentiation of the experts' subordinate categories found in Experiment 1, it is possible that a shift in object naming might occur with expertise. Experts may tend to categorize objects in the domain of expertise at a more specific level of abstraction and subsequently, experts should use subordinate-level names morc often than novices for identification. For example, the expert birdwatcher might use the subordinate-level name "robin" rather than the basic level name "bird" when identifying this animal. For object classifications outside the domain of expertise, subjects should use basic-level names for identification (e.g., the expert birder should use the name "dog" when shown a picture of a beagle). To test this hypothesis, dog and bird experts were asked to name pictures of four dog and bird exemplars as quickly as possible.

\section{Method}

Subjects. The subjects were 12 dog experts recruited from a local dog organization and 12 bird experts who were members of a local birdwatching association. Nine of the dog experts and eight of the bird experts participated in Experiment 1. All subjects had a minimum of 10 years of experience in their area of expertise. The group of dog experts consisted of nine women and three men ranging in age from 39 to 70 years with a mean age of 53 years. The group of hird experts consisted of four women and eight men ranging in age from 38 to 76 years with a mean age of 53 years. None of the subjects had expertise in both the dog and bird domains. Subjects were paid for their participation.

Stimuli. Picture stimuli consisted of 86 black-and-white drawings and photographs of common objects mounted on $15.4 \times 12.6 \mathrm{~cm}$ white index cards. To minimize response bias, 
the target dog and bird pictures were embedded in a larger set of 78 filler pictures drawn from artifactual and natural kind categories. The artifactual categories were musical instrument, sports equipment, vehicle, food, furniture, tool, clothing, foot gear, jewelry, office equipment, cooking utensil, kitchen appliance, and home electronics. The natural categories were dog, bird, fish, insect, tree, flower, vegetable, fruit, four-legged animal, and famous person. The pictures of the four bird exemplars (robin, sparrow, jay, and cardinal) were taken from a book on bird identification (Zim, 1949). Robin, sparrow, jay, and cardinal were among the 10 most frequently mentioned birds in the Battig and Montague (1969) category norms. The pictures of the four dog exemplars (German shepherd, Doberman pinscher, beagle, cocker spaniel) were taken from an encyclopedia. German shepherd, Doberman pinscher, beagle, and cocker spaniel were among the $\mathbf{2 0}$ most frequently occurring dogs as determined by the American Kennel Club's list of registered dogs for 1984.

Procedure. The subject was seated at a table directly across from the experimenter. Subjects were instructed that they would see a series of pictures depicting common everyday objects. Their task was "to say the word that names the object as quickly as possible." The experimenter presented each picture one at a time and recorded the category level (i.e., superordinate, basic, or subordinate) of each response. For the target pictures in the domain of expertise, the experimenter also noted the order of appearance (i.e., first, second, third, or fourth position). Pictures were presented at a rate of approximately one picture every 2 s. The order of presentation was randomized across subjects with the restriction that consecutive pictures were drawn from different superordinate categories.

According to Rosch et al., novices use basic-level names when identifying objects because this is the most useful level of abstraction for referring to objects. However, the use of basic-level names could also be due to lack of knowledge about subordinate-level categories. For example, dog experts might use the label "bird" rather than "sparrow" because they do not know the features that distinguish sparrows from other birds. To assess the possibility that novices were unfamiliar with the subordinate-level categories, after the naming portion of the experiment, subjects were asked to identify the four pictures from the novice knowledge domain using their subordinate names. Any picture that could not be identified at the subordinate level was excluded from the main analysis.

\section{Results and Discussion}

None of the pictures was identified with a superordinate term. The comparison of primary interest was the percentage of trials in which basic versus subordinate level names were used to identify objects in the domain of expertise. On $57 \%$ of the trials, pictures of objects from the expert domain were identified with subordinate-level names and on $43 \%$ of the trials, they were identified with basic-level names. The difference in proportions between the basic-level and subordinate-level names was not significant, $\chi^{2}(1)=2.04, n s$. Rather than consistently applying the basiclevel name, expert subjects frequently used subordinate-level names. The likelihood of using a subordinate-level name was not affected by the target picture's order of appearance, $\chi^{2}(3)=2.76, n s$.

For identifying pictures of objects in the novice domain, subjects used basic-level names on $76 \%$ of the trials and subordinate-level names on $21 \%$ of the trials (3\% of the trials were omitted from the analysis because pictures could not be identified with the appropriate subordinate-level names). The difference between the occurrence of basic-level and subor- 
dinate-level names was significant, $\chi^{2}(1)=26.50, p<.001$. Thus, our results for the novice domain replicate past research showing that subjects tend to use basic-level names for identifying objects (Jolicoeur et al., 1984; Rosch et al., 1976; Segui \& Fraisse, 1968).

Separate analyses performed on the dog and bird experts revealed a difference in the naming patterns between the two types of experts. As shown in Fig. 2, bird experts identified bird pictures with subordinatelevel names on $74 \%$ of the trials and on $26 \%$ of the trials, they choose basic-level names, $\chi^{2}(1)=12.00, p<.001$. Thus, for identifying objects from their domain of expertise, bird experts preferred subordinate-level names over basic-level names. On the other hand, although dog experts used subordinate category labels more frequently than novices, they did not show a distinct preference for either basic- or subordinate-level labels. On $60 \%$ of the trials they used the basic-level label and on $40 \%$ of the trials they used the subordinate-level label, $\chi^{2}(1)=2.08$, ns. This difference between dog and bird experts in naming performance may be due to the different skills that are emphasized for acquiring expertise in these two fields. Almost by definition, an expert birdwatcher is a person who can make fast and accurate perceptual identifications at specific levels of abstraction. In contrast, dog expertise tends to take the form of experience in the handling, training, grooming, and breeding of one or two particular breeds. In their study of dog experts, Diamond and Carey (1986) found that subjects showed effects of expertise only for those breeds of dog in which they specialized. As it turned out, only one of our experts had prior direct contact with the breeds used in the naming experiment. Our point is not that dog experts know about only one or two

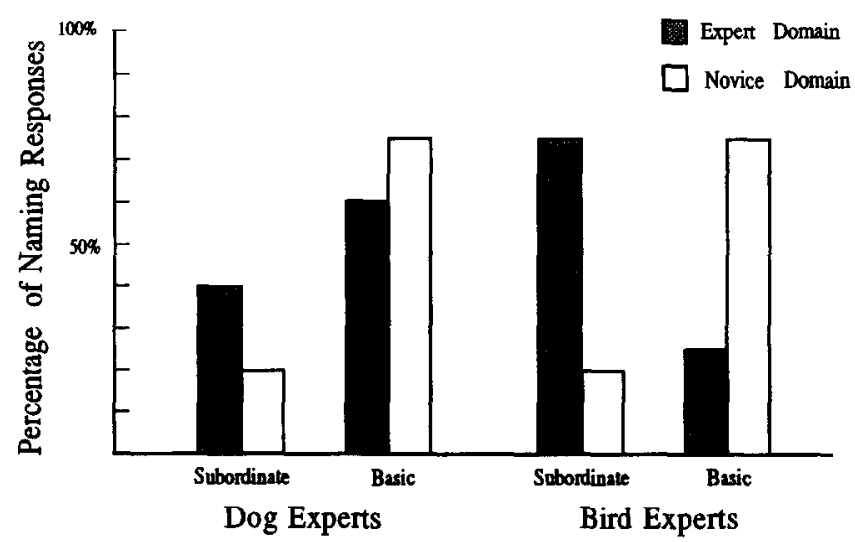

FIG. 2. Percentage of pictures identified with subordinate-level and basic-level names as a function of knowledge domain (expert and novice) and expert type (dog experts and bird experts). 
breeds of dogs (the results of Experiment 1 indicate that they know more than novices about the breeds used in this research), but that identifying specific breeds of dogs is less central to the activities of dog experts than identifying specific species of birds is to the activities of bird experts.

In summary, the results from the naming study demonstrate that expert subjects frequently use subordinate-level names for identifying objects in their domain of expertise. This result can be contrasted to the primary use of basic-level names by novices (Jolicoeur et al., 1984; Rosch et al., 1976; Segui \& Fraisse, 1968). Taken together with the findings from Experiment 1 , these results show that experts are more informed about the specific features that distinguish exemplars at the subordinate level, and they have ready access to this information for the purpose of object naming. The accessibility of subordinate-level versus basic-level representations is further examined in Experiment 3.

\section{EXPERIMENT 3}

\section{Category Verification}

Based on their analysis of category structure, Rosch et al. (1976) predicted that when an object is first perceived, it will be categorized at the basic level of abstraction. In the literature, the initial contact between the object percept and semantic representation has been referred to as primal access (Biederman, 1987) or entry point (Jolicoeur et al., 1984). To test the hypothesis that the "first cuts" in object categorization are made at the basic level, Rosch et al. (1976) used a category verification paradigm. In their experiment, subjects first heard a category label (superordinate, basic, or subordinate) and then were asked to indicate if a picture was an exemplar of the category. The results showed that subjects were faster to categorize objects at the basic level than at the superordinate or subordinate levels.

Experiments using artificial categories and controlling for linguistic differences between categories at different levels of abstraction indicate that the basic-level advantage in picture categorization is not due to the fact that basic-level names are shorter and used more frequently than words for superordinate or subordinate categories (Murphy \& Smith, 1982). Instead the basic-level advantage seems to be related to the differentiation of basic-level categories. According to Murphy and Brownell (1985), category differentiation is a function of distinctiveness ("how dissimilar a category is to its contrast categories", p. 71) and specificity ("how specific, informative, or narrowly defined a category concept is", p. 71). On one hand, superordinate-level categories are highly distinctive, but not very informative, and on the other hand, subordinate-level categories are highly informative, but are not very distinctive. Thus, with respect to differentiation, the intermediate basic-level categories represent the op- 
timal level of categorization because they are both specific (as compared to superordinate-level categories) and distinctive (as compared to subordinate-level categories).

Murphy and Brownell (1985) (also see Jolicoeur et al., 1984) tested the differentiation hypothesis with a category verification task in which the typicality of the stimuli was varied. Although typical subordinate exemplars of a basic category are not very distinctive (e.g., robins look a lot like other types of birds), atypical subordinate exemplars are distinctive because they do not share many features with other exemplars of the basic-level category (e.g., penguins do not look like other types of birds). Subjects should be faster to categorize atypical exemplars at the subordinate level because they are highly differentiated (i.e., they are both specific and distinctive), and slower to categorize them at the basic level because they are atypical. Hence, subjects should be faster to verify that an object is a penguin than that it is a bird. This prediction was confirmed by the results of Murphy and Brownell (1985).

Given that experts, unlike novices, know many features of typical subordinate categories, and the majority of features they list for one category (e.g., robin) do not overlap with features listed for other subordinate categories (e.g., crow), it is possible that expertise as well as typicality contributes to category differentiation. Thus, according to the predictions of the differentiation hypothesis, experts should be able to categorize objects as fast at the subordinate level as at the basic level because their basic- and subordinate-level categories are equally differentiated. For example, a bird expert would be as fast to verify that an object is a robin as to verify that the same object is a bird. In the novice domain, we would expect the verification times to be fastest at the basic level.

\section{Method}

Subjects. The subjects were 12 dog experts recruited from a local dog organization and 12 bird experts who were members of a local birdwatching association. All the dog and bird experts participated in either Experiments 1 or 2 . The group of dog experts consisted of ten women and two men ranging in age from 40 to 70 years with a mean age of 54 years. The group of bird experts consisted of five women and seven men ranging in age from 32 to 76 years with a mean age of 51 years. Subjects were paid for their participation.

Materials. The picture stimuli consisted of eight colored dog drawings taken from the Spotter's Guide to Dogs (Glover, 1976) and eight colored bird drawings from Peterson's First Guide to Birds (1985). An additional 16 pictures depicting various trees and rock formations were used as foil stimuli. Each picture was mounted on a gray $8 \times 11 \frac{1}{2}$ " sheet of paper and photographed using 34-mm color slide film. The eight bird exemplars (robin, crow, sparrow, hawk, jay, cardinal, oriole, and pigeon) were selected from Battig and Montague's (1969) category norms with the restriction that none of the exemplars included a color word in its name. Seven of the eight bird exemplars were among the 10 most frequently mentioned birds in the Battig and Montague study; oriole was the 13th most frequently mentioned bird. The eight dog exemplars (German shepherd, Doberman pinscher, beagle, chow chow, schnauzer, poodle, collie, and dachshund) were among the 20 
most frequently occurring dogs as determined by the American Kennel Club's list of registered dogs for 1984. None of the dog exemplars included a color word in its name.

Procedure. At the beginning of the experiment, subjects read a set of written instructions that explained the procedure for the categorization task and were given a list of the 16 target exemplars that they were about to be shown. Although other studies have shown no effects of familiarization on reaction times in picture verification tasks (Biederman \& Ju, 1988), there was concern that experts may be unduly conservative when making judgments in their expert domain. For example, the dog expert may hesitate when responding affirmatively to the category name of "collie" because of its close physical resemblance to the "sheltie" dog. Thus, familiarizing subjects beforehand with the range of exemplars helped to limit the possible alternatives under consideration.

Subjects were given 10 practice trials. The reaction-time paradigm for the experiment was similar to that used in previous category verification experiments (Murphy \& Brownell 1985; Rosch et al., 1976). At the beginning of each trial, a row of plus signs appeared on the computer monitor which served as a ready signal. After a 1-s interval, the ready signal was replaced by a category name. A picture appeared on a projection screen directly in front of the subject $2.5 \mathrm{~s}$ following the onset of the category word. According to Murphy and Brownell (1985), a relatively long stimulus onset asychrony (2.5 s) is needed to ensure that subjects have sufficient time to read and comprehend the typically longer subordinate category level words. If the picture matched the word, the subject was instructed to press the "true" key; otherwise they were to respond by pressing the "false" key. Subjects used the index finger of their dominant hand and they were instructed to respond as quickly as possible. Both word label and picture remained visible until the subject responded. At the halfway point of the experiment, subjects were given a 2-min rest period.

Each of the $16 \mathrm{dog}$ and bird pictures was shown six times. For the three TRUE trials, the category name matched the picture at the superordinate, basic, and subordinate levels. For each picture's three FALSE trials, the category names contrasted with the target category at the same level of abstraction. Consequently, subordinate foils shared the same basic category as the target (e.g., a picture of a robin was paired with "crow"), basic-level foils shared the same superordinate category as the target (e.g., a picture of a bird was paired with "dog") and superordinate foils were taken from another superordinate category (e.g., a picture of an animal was paired with "plant"). To minimize response confusion due to the high degree of visual similarity shared by subordinate exemplars (Murphy \& Brownell, 1985), subordinate pairings were determined by three independent judges who were instructed to maximize the visual contrast between target pictures. ${ }^{3}$ Thirty-two additional trials of filler pictures (e.g., trees and rocks) were shown to prevent response bias to superordinate foils (i.e.. automatically responding FALSE to the "plant" foil). Half of the filler trials were TRUE and half were FALSE. Including the 96 target and 32 filler trials, there were a total of 128 trials. The presentation of the stimulus trials was randomized across subjects.

\footnotetext{
${ }^{3}$ Reaction times of subordinate level categorizations can be manipulated by varying the category from which target pictures' foil is taken (Murphy \& Brownell, 1985). For example, if the picture for the subordinate level foil is selected from a different superordinate category, subjects could readjust their decision criterion and respond very quickly at the subordinate level. On the other hand, subjects will use a highly conservative subordinate level decision criterion when subordinate level foils are very similar in visual appearance (e.g., retriever versus setter). Therefore, an intermediate subordinate level decision criterion was achieved by selecting subordinate level foils that matched the target's category name at the same level of abstraction, but were different in visual appearance.
} 


\section{Results and Discussion}

Comparison of error rates showed that there was no speed-accuracy trade-off. For TRUE responses in the domain of expertise, error rates were 5,3 , and $2 \%$ for superordinate, basic, and subordinate levels, respectively. The percentages of errors in the novice area for true responses were higher at 13, 4, and $10 \%$ for superordinate, basic, and subordinate decisions, accordingly. For FALSE trials, the error rates in the expert domain were 10,12 , and $7 \%$ as compared with 7,4 , and $11 \%$ in the novice domain for superordinate, basic, and subordinate categorizations, respectively.

An analysis of variance test was performed for the TRUE trials with expert type (bird, dog) as a between-group factor and category level (superordinate, basic, subordinate) and knowledge domain (expert and novice) as within-subject factors. The between-group factor of expert type was not significant, $F(1,22)=.89, M S e=167,417$. The main effect of knowledge domain was significant, $F(1,22)=21.70, M S e=206,570, p<$ .001 . Subjects were faster to make category judgments in their area of expertise than outside their domain of expertise. The difference in response times for the levels of categorization was also significant, $F(2,44)$ $=8.03, M S e=90,494, p<.001$. The predicted interaction between category level (superordinate, basic, subordinate) and knowledge domain (expert, novice) was significant, $F(2,44)=11.91, M S e=62,286, p<$ .001 . As shown in Fig. 3, experts were as fast to categorize at the subordinate level in their expert domain as they were to categorize at the basic level. In the novice domain, their reaction times showed the more

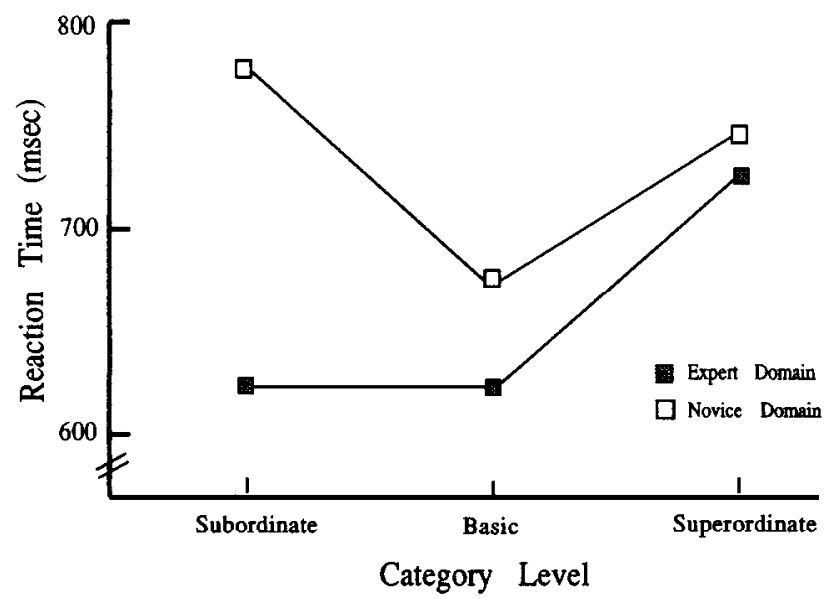

FIg. 3. Mean reaction times for TRUE responses as a function of knowledge domain (expert and novice) and category level (subordinate, basic, and superordinate). 
typical pattern of response; that is, reaction times were faster at the basic level than at the superordinate or the subordinate level. Table 2 shows the separate reaction times for true responses as a function of category level, knowledge domain, and expert type.

Direct comparisons between TRUE judgments showed that subordinate-level judgments in the expert domain were significantly faster than subordinate judgments in the novice domain, $t(23)=5.66, p<.001$. In category verification, expertise seems to have its most profound effects on the subordinate categories in the expert domain. This finding is consistent with the result from Experiment 1 where experts listed significantly more distinctive features for subordinate categories in the domain of expertise than for subordinate categories in the novice domain. While the related comparison between reaction times for the expert-basic and novice-basic categorizations was also significant, $t(23)=2.12, p<.05$, this difference was carried almost exclusively by the dog experts. As shown in Table 2, the dog experts were much faster to verify that a picture of a dog was indeed a dog than to verify that a picture of a bird was a bird. The bird experts showed little difference in basic-level categorization times across the two knowledge domains. This difference between dog and bird experts is consistent with the results of the naming experiment showing that dog experts use the basic-level name "dog" more often than bird experts used the basic-level name "bird." The reaction time difference in the category verification study may be related to the differences between dog and bird experts discussed earlier. Clearly, the nature of expertise may vary as a function of domain in ways that can affect the process of categorization.

An analysis of variance test was also performed for the FALSE trials with expert type (bird, dog) as a between-subjects factor and category level (superordinate, basic, subordinate) and knowledge domain (expert and novice) as within-subject factors. The group difference between the

TABLE 2

Reaction Times for TRUE Responses as a Function of Category Level, Knowledge Domain, and Expert Type

\begin{tabular}{lccc}
\hline & \multicolumn{3}{c}{ Category level } \\
\cline { 3 - 4 } Knowledge domain & Subordinate & Basic & Superordinate \\
\hline $\begin{array}{l}\text { Dog experts } \\
\text { Dog domain }\end{array}$ & $608 \mathrm{~ms}$ & $630 \mathrm{~ms}$ & $753 \mathrm{~ms}$ \\
$\begin{array}{c}\text { Bird domain } \\
\text { Bird experts }\end{array}$ & $824 \mathrm{~ms}$ & $737 \mathrm{~ms}$ & $826 \mathrm{~ms}$ \\
Dog domain & $731 \mathrm{~ms}$ & $618 \mathrm{~ms}$ & $665 \mathrm{~ms}$ \\
Bird domain & $635 \mathrm{~ms}$ & $616 \mathrm{~ms}$ & $704 \mathrm{~ms}$ \\
\hline
\end{tabular}


bird and dog experts approached significant levels, $F(1,22)=3.36, M S e$ $=633,218, p<.09$, demonstrating that on FALSE trials, the bird experts were faster than the dog experts. The main effect of knowledge domain also approached significant levels, $F(1,22)=3.73, M S e=45,546, p<$ .07. The critical interaction between category level and knowledge domain was significant, $F(2,44)=14.53, M S e=89,461, p<.001$. As shown in Fig. 4, comparisons of the combined groups' false responses showed that in the domain of expertise, subordinate-level responses were significantly faster than basic-level categorizations (e.g., a bird expert judging that a picture of a sparrow was not a "robin" was faster than judging that the same picture was not a " $\operatorname{dog}$ "), $t(23)=2.53, p<.02$. However, in the domain outside their realm of expertise, basic-level judgments were significantly faster than subordinate-level judgments, $t(23)=5.61, p<.001$. Table 3 shows the reaction times for FALSE responses as a function of category level, knowledge domain, and expert type.

Overall, the pattern of results from this experiment suggests that expertise enhances the speed at which subordinate-level categories are accessed, making them at least as accessible as basic level categories. Thus, our results support the claim that category differentiation plays an important role in object recognition (Murphy \& Brownell, 1985). An interesting question to consider is why experts were not faster to verify category membership at the subordinate level than at the basic level. Rosch et al.'s (1976) comments about the location of the basic level possibly differing for experts and novices can be interpreted as predicting that the bird experts, for example, would be faster to identify objects at the level of

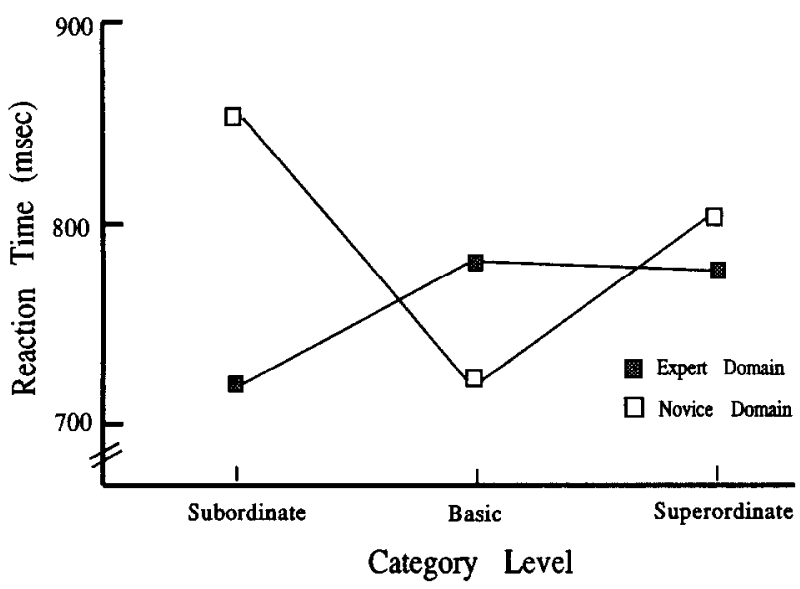

Fig. 4. Mean reaction times for FALSE responses as a function of knowledge domain (expert and novice) and category level (subordinate, basic, and superordinate). 
TABLE 3

Reaction Times for FALSE Responses as a Function of Category Level, Knowledge Domain and Expert Type

\begin{tabular}{lccc}
\hline & \multicolumn{3}{c}{ Category level } \\
\cline { 2 - 4 } Knowledge domain & Subordinate & Basic & Superordinate \\
\hline $\begin{array}{l}\text { Dog experts } \\
\text { Dog domain }\end{array}$ & $756 \mathrm{~ms}$ & $811 \mathrm{~ms}$ & $823 \mathrm{~ms}$ \\
$\begin{array}{l}\text { Bird domain } \\
\text { Bird experts }\end{array}$ & $955 \mathrm{~ms}$ & $795 \mathrm{~ms}$ & $930 \mathrm{~ms}$ \\
Dog domain & & & $674 \mathrm{~ms}$ \\
Bird domain & $755 \mathrm{~ms}$ & $671 \mathrm{~ms}$ & $721 \mathrm{~ms}$ \\
\hline
\end{tabular}

"robin" than at the level of "bird." Despitc Rosch et al.'s (1976) speculation, it seems unlikely to us that experts would be faster to categorize typical exemplars at the subordinate level than at the basic level. Others (Murphy \& Smith, 1982) have argued that a more stringent criterion is adopted for subordinate-level judgments than for basic-level judgments, presumably because typical subordinate-level categories share greater feature overlap with their contrast categories than basic-level categories. The addition of differentiating subordinate features as a result of expertise may offset the initial advantage held by the basic level, but cannot completely eliminate its categorization status. Moreover, we (Experiment 1) as well as Tversky and Hemenway (1984) have shown that whereas basiclevel categories can be differentiated from their contrast categories on the basis of their "part" features (e.g., beak, feathers), subordinate-level categories are distinguishable more on the basis of their "modified part" features (e.g., elongated beak, brown feathers). Therefore, a more quantitative visual analysis may be needed for distinguishing subordinate-level categories from each other than is required for distinguishing between basic-level categories. Consistent with the interpretation that subordinate-level categories require a finer grain of visual analysis, Jolicoeur et al. (1984) found that subordinate-level categorizations were more negatively affected by limited perceptual encoding time than were basic-level categorizations.

There was one exception to our finding that experts were as fast at the basic level as at the subordinate level. On FALSE trials involving pictures in the domain of expertise, subjects were faster to disconfirm category membership at the subordinate level than at the basic level. The interpretation of this finding is complicated by the fact that the subordinate-level trials involved within-domain judgments while the basic-level trials involved between-domain judgments (e.g., a basic-level category word from the novice domain and a picture from the expert domain). Although 
the finding of faster responses for subordinate FALSE trials is interesting, we do not think it provides sufficient evidence by itself to support the claim that, for experts, subordinate-level categories are more accessible than basic-level categories.

\section{GENERAL DISCUSSION}

While Rosch et al. (1976) claimed that the privileged status of the basiclevel category is a reflection of the general characteristics of the human perceiver and inherent structure of objects in the world, they also acknowledged the possible contribution of individual differences in knowledge to the organization of human categories: "Different amounts of knowledge about objects can change the classification scheme. Thus, experts in some domain of knowledge can make use of attributes that are ignored by the average person" (p. 430). The present experiments were designed to address this speculation. In the first experiment, it was found that in novice domains, subjects listed the greatest number of new features for basic-level categories, but, in their domain of expertise, they listed equivalent numbers of new features for subordinate-level and basiclevel categories. The implications of increased knowledge at the subordinate level were demonstrated in Experiments 2 and 3. In Experiment 2, subjects used the subordinate-level name as frequently as the basic-level name for identifying objects in their domain of expertise. In Experiment 3 , it was found that experts were as fast to verify category membership at the subordinate level as at the basic level.

\section{Implications for What Is Meant by the "Basic Level"}

Discussions of conceptual hierarchies often suggest that the basic level can be defined as the level which is the most psychologically fundamental. For example, the basic level is claimed to be the first level of categorization for object recognition, objects are typically identified at the basic level, and names for basic categories are the first words acquired by children. According to this view, it is possible to describe basic-level categories at a more specific level of abstraction for one group of individuals and at a more inclusive level for another. In other words, one might hypothesize a downward shift in the basic level or the creation of a second more specific basic level in a classification hierarchy as a function of expertise. The downward shift hypothesis is implicit in Rosch et al.'s (1976) speculation that the basic level for a fish expert would be the level of trout, bass, and salmon; categories generally considered to be at the subordinate level for novice populations. Rosch et al. (1976) also considered the possibility of experts having hierarchies with two or more basic levels, and discussed which types of hierarchies would allow the creation of multiple basic levels. 
Although a definition of the basic level in terms of psychological preference has some advantages (e.g., the notion of basic level is not limited to concrete objects like dogs and chairs, but can be extended to other types of categories such as emotions and personality traits (John, Hampson, \& Goldberg, 1990; Shaver, Schwartz, Kirson, \& O'Connor, 1987)), the basic level is often conceptualized quite differently (Jolicoeur et al., 1984; Mervis, 1987; Mervis \& Crisafi, 1982; Murphy \& Smith, 1982; Rosch, 1978). Rosch et al. originally used four converging operational definitions of the basic level: (1) the level which maximizes information gain as indexed by subjects' attribute lists; (2) the most inclusive level at which objects elicit highly similar sequences of motor movements; (3) the most inclusive level at which the shapes of object are very similar as indexed by the ratio of overlapping to nonoverlapping areas for normalized pictures of category exemplars; and (4) the most inclusive level at which an averaged shape of an object is identifiable. Because of the emphasis on "most inclusive" in these definitions, we interpret them collectively as based primarily on perceived structure in the world and as inconsistent with the idea of a downward shift in the location of the basic level as a function of expertise. Definitions based on perceived structure in the world make the location of the basic level relatively independent of the effects of the domain-specific expertise. Although the subordinatelevel categories of the experts are more differentiated than the novices', the basic level remains the most inclusive at which objects look alike. For example, even though the characteristics that distinguish breeds of dogs are better known to dog experts, for experts (and for novices as well), the level of "dog" would still be the most general level at which these animals would share similar overall shapes.

Our work with experts indicates that it is important not to assume that the psychological definition and the "perceived structure" definition of the basic-level category always converge on the same level of abstraction. Past research has demonstrated such a convergence because the subjects have been selected from novice populations in a single culture. However, our experiments with experts show that extensive knowledge in a domain may result in categories at the level of "collie" and "robin" sharing some of the psychological advantages usually attributed solely to categories at the level of "dog" and "bird." In keeping with Rosch et al.'s operational definition of the basic level, we interpret our results not as the construction of a second basic level, but as an increase in the accessibility of the subordinate level as a function of expertise.

\section{Implications for the Process of Categorization}

According to Rosch et al. (1976), objects are first identified at the basic level. Subsequent categorizations at the superordinate level involve retrieval of semantic information and categorizations at the subordinate 
level require additional perceptual analysis (Jolicoeur et al., 1984). The basic first hypothesis requires that basic-level categorizations are always faster than superordinate-level or subordinate-level categorizations because perceptual input accesses semantic memory initially at the basic level. However, counter to the predictions of the basic first hypothesis, we found in Experiment 3 that expert subjects made subordinate-level categorizations as quickly as basic-level categorizations, suggesting that basic-level and subordinate-level categorizations can be performed independently of each other. Other studies have challenged the basic first hypothesis by showing that atypical exemplars of a basic-level category (e.g., racing car) are categorized faster at the subordinate level than at the basic level (Murphy \& Brownell, 1985; Jolicoeur et al., 1984). Recently, Murphy and Wisniewski (1989) have also shown that superordinate-level categorizations can be as fast as basic-level categorizations when objects are placed in the appropriate contextual scene. Thus, the converging evidence shows that, contrary to the basic first hypothesis, people do not automatically identify objects at the basic level before making categorizations at the other levels of abstraction.

Murphy and Brownell's (1985) differentiation hypothesis seems to provide a more parsimonious account of the empirical findings. The differentiation hypothesis maintains that the accessibility of a category is a function of specificity and distinctiveness, taken together as the degree of category differentiation. The more differentiated the category, the more easily it can be accessed. This hypothesis is consistent with the general finding that objects are usually categorized fastest at the basic level (Rosch et al., 1976), and with the exceptions to the basic-level finding: (1) when an object is an atypical exemplar of a basic-level category (i.e., its subordinate is more distinctive than most subordinates from the same basic level), it is categorized faster at the subordinate level than at the basic level, and (2) when people are particularly knowledgeable about the features that distinguish subordinate categories in a domain (i.e., they become experts), their subordinate-level categorizations are as fast as their basic-level categorizations.

\section{Variability in Expertise across Individuals and across Cultures}

The exact nature of the difference between an expert and a novice is bound to be affected by characteristics of the expert domain (e.g., the type of activity that the expert participates in, the goals or reasons for the expert knowledge, important aspects of the objects in the domain). The domain of birds is quite similar to the domain of dogs when one considers the wide range of topics on which one could become an expert (e.g., chess, automechanics, stamps, flowers, etc.), yet dog experts and bird experts differed in some respects. For example, bird experts showed a clear preference for naming birds at the subordinate level while dog ex- 
perts used both subordinate- and basic-level names for identifying dogs. The differences between experts in diverse domains are interesting topics for research, but the main point of the present investigation was to demonstrate the increased psychological importance of more specific categories as a function of expert knowledge. We believe this is a general finding with broad applications. For example, research on social stereotypes indicates that when a person is well-known, their membership in a social category (e.g., Asian, black) tends not to be invoked because they are encoded at a more specific level of abstraction (see Rothbart \& John, 1985).

We also think that our results on the effects of expertise are not limited to people who like our subjects have devoted many years of their life to gaining knowledge and experience in a particular domain. Expertise does not have to span an entire domain. Instead, it could be quite narrow in scope, perhaps limited to a single subordinate category. For example, a person who owns a collie and spends a lot of time with the dog could be considered a "collie expert." Such a person might be aware of the distinguishing features of collies, but know very little about distinctive properties of other breeds of dogs. Presumably, this person would refer to collies by their subordinate-level name, but refer to other breeds of dogs simply as "dogs." We suspect such specificity in the level of categorization is commonplace, and consequently, there may be considerable unevenness in the way an individual categorizes objects in a single domain. Rosch et al. (1976) considered this possibility, giving the example of an antique furniture dealer "for whom Chippendale and Hepplewhite chairs are the basic level objects, but for whom kitchen and living room chairs, in the average house, are as undifferentiated as for our subjects" (p. 432). ${ }^{4}$

In addition to individual differences in the way particular objects are categorized as a function of idiosyncratic life experiences, it is also possible to suggest that differences in the way objects are categorized would exist hetween members of different population groups. Within a given culture, there may be a typical level of expertise with respect to a particular domain, determined by the relative importance of the domain for the culture. However, the importance of a given domain may vary crossculturally, with more specific levels of categorization being associated with cultures in which the domain is more important. Evidence drawn from cross-cultural studies is consistent with this idea. The basic level, as defined by the most inclusive level at which objects share perceptual features, is not necessarily the level at which objects are usually categorized in nonwestern socicties. For example, Dougherty (1978) found that the Tzeltal Mayan people identify common plants and animals at the

${ }^{4}$ In this example, Rosch et al. (1976) use the psychological definition of the basic level rather than the definition based upon perceived structure in the world. 
folk-genera level (e.g., oak) rather than at the life-form level (e.g., tree). In a related study, Stross (1973) found that Tzeltal children first learn the names of plants corresponding to our subordinate-level categories and later on, acquire the more inclusive basic-level names. Presumably, the pragmatic concerns of an agrarian society make the more specific categorization of plants and animals desirable (Cruse, 1975).

In conclusion, this research emphasizes diversity in conceptual structure as a function of expertise and is consistent with the views of many researchers (Jolicoeur et al., 1984; Murphy \& Medin, 1985; Murphy \& Smith, 1982; Rosch et al., 1976) concerning the role that world knowledge plays in shaping object categorization. By emphasizing the importance of world knowledge, we do not mean to suggest that stimulus structure plays no role in the categorization process. However, while the external environment and the human perceptual system impose certain constraints, human categorization appears to be continually reshaped and altered by learning and experience.

\section{REFERENCES}

Barsalou, L. W. (1985). Ideals, central tendency, and frequency of instantiation as determinants of graded structure in categories. Journal of Experimental Psychology: Learning, Memory, and Cognition, 11, 629-653.

Battig, W. F., \& Montague, W. E. (1969). Category norms for verbal items in 56 categories: A replication and extension of the Connecticut category norms. Journal of Experimental Psychology Monograph, 80, 1-46.

Biederman, I. (1987). Recognition-by-components: A theory of human image understanding. Psychological Review, 94, 115-147.

Biederman, I., \& Ju, G. (1988). Surface versus edge-based determinants of visual recognition. Cognitive Psychology, 20, 38-64.

Biederman, I., \& Shiffrar, M. (1988). Sexing day-old chicks: A case study and expert systems analysis of a difficult perceptual-learning task. Journal of Experimental Psychology: Learning, Memory, and Cognition, 13, 640-645.

Brown, R. (1958). How should a thing be called? Psychological Review, 65, 14-21.

Carey, S. (1985). Conceptual change in childhood. Cambridge, MA: MIT Press.

Chase, W. G., \& Simon, H. A. (1973). Perception in chess. Cognitive Psychology, 4, 55-81.

Chi, M. T. H., Feltovich, P. J., \& Glaser, R. (1981). Categorization and representation of physics problems by experts and novices. Cognitive Science, 5, 121-152.

Cruse, D. A. (1975). The pragmatics of lexical specificity. Journal of Linguistics, 13, 153164.

Diamond, R., \& Carey, S. (1986). Why faces are and are not special: An effect of expertise. Journal of Experimental Psychology: General, 115, 107-117.

Dougherty, J. W. D. (1978). Salience and relativity in classification. American Ethnologist, $5,66-80$.

Glover, H. (1976). Spotter's guide to dogs. New York: Smith Publishers.

John, O. P., Hampson, S. E., \& Goldberg, L. R. (1990). The basic level in personality-trait hierarchies: Studies of trait use and accessibility in different contexts. Journal of Personality and Social Psychology, 60, 348-361.

Jolicoeur, P., Gluck, M. A., \& Kosslyn, S. M. (1984). Picture and names: Making the connection. Cognitive Psychology, 16, 243-275. 
Lakoff, G. (1987). Women, fire and dangerous things: What categories tell us about the nature of thought. Chicago: University of Chicago Press.

Malt, B. C., \& Smith, E. E. (1984). Correlated properties in natural categories. Journal of Verbal Learning and Verbal Behavior, 23, 250-269.

Medin, D. L., \& Ortony, A. (1989). Psychological essentialism. In S. Vosniadou \& A. Ortony (Eds.), Similarity and analogical reasoning (pp. 179-195). New York: Cambridge University Press.

Mervis, C. B. (1987). Child-basic object categories and early lexical development. In U. Neisser (Ed.), Concepts and conceptual development: Ecological and intellectual factors in categorization (pp. 201-233). Cambridge: Cambridge University Press.

Mervis, C. B., \& Crisafi, M. A. (1982). Order of acquisition of subordinate-, basic-, and superordinate-level categories. Child Development, 53, 258-266.

Mervis, C. B., \& Rosch E. (1981). Categorization of natural objects. Annual Review of Psychology, 32, 89-115.

Murphy, G. L., \& Brownell, H. H. (1985). Category differentiation in object recognition: Typicality constraints on the basic category advantage. Journal of Experimental Psychology: Learning, Memory and Cognition, 11, 70-84.

Murphy, G. L., \& Medin, D. L. (1985). The role of theories in conceptual coherence. Psychological Review, 92, 289-316.

Murphy, G. L., \& Smith, E. E. (1982). Basic level superiority in picture categorization. Journal of Verbal Learning and Verbal Behavior, 21, 1-20.

Murphy, G. L., \& Wisniewski, E. J. (1989). Categorizing objects in isolation and in scenes: What a superordinate is good for. Journal of Experimental Psychology: Learning, Memory, and Cognition, 15, 572-586.

Murphy, G. L., \& Wright, J. C. (1984). Changes in conceptual structure with expertise: Differences between real-world experts and novices. Journal of Experimental Psychology: Learning, Memory and Cognition, 10, 144-155.

Neisser, U. (1987). Introduction: The ecological and intellectual bases of categorization. In $\mathrm{U}$. Neisser (Ed.), Concepts and conceptual development: Ecological and intellectual factors in categorization (pp. 1-10). Cambridge: Cambridge University Press.

Peterson, R. T. (1985). First field guide to birds. Boston: Houghton Mifflin.

Rosch, E. (1978). Principles of categorization. In E. Rosch \& B. B. Lloyd (Eds.), Cognition and categorization (pp. 27-48). Hillsdale, NJ: Erlbaum.

Rosch, E., Mervis, C. B., Gray, W., Johnson, D., \& Boyes-Braem, P. (1976). Basic objects in natural categories. Cognitive Psychology, 8, 382-439.

Rothbart, M., \& John, O. P. (1985). Social categories and behavioral episodes: A cognitive analysis of the effects of intergroup contact. Journal of Social Issues, 41, 81-104.

Schvaneveldt, R. W., Durso, F. T., Goldsmith, T. E., Breen, T. J., \& Cooke, N. J. (1985). Measuring the structure of expertise. International Journal of Man-Machine Studies, 23, 699-728.

Segui, J., \& Fraisse, P. (1968). Le temps de reaction verbale. III Responses specifiques et responses icategorielles a des stimulus objects. L'Annee psychologique, 68, 69-82.

Shaver, P., Schwartz, J., Kirson, D., \& O’Conner, C. (1987). Emotion knowledge: Further explanation of a prototype approach. Journal of Personality and Social Psychology, 52 , 1061-1086.

Stross, B. (1973). Acquisition of botanical terminology by Tzeltal children. In M. Edmonson (Ed.), Meaning in Mayan language (pp. 109-141). The Hague: Mouton.

Tversky, B., \& Hemenway, K. (1984). Objects, parts and categories. Journal of Experimental Psychology: General, 113, 169-193.

Zim, H. (1949). Birds. New York: Simon \& Schuster.

(Accepted September 11, 1990) 